\title{
Efectos de cuatro niveles dietéticos de harina de semilla de chocho (Lupinus mutabilis) sobre parámetros productivos, desarrollo intestinal y valores hematológicos en pavos de ocho semanas
}

\author{
Effects of four dietary levels of lupine seed meal (Lupinus mutabilis) on \\ productive parameters, intestinal development and haematological values \\ in eight-week-old turkeys
}

\author{
Manuel Paredes A. ${ }^{1,2}$, Enilyn de la Flor M. ${ }^{1}$, José Mantilla G. ${ }^{1}$
}

\section{Resumen}

El efecto de cuatro niveles dietéticos de la harina de semilla de chocho (Lupinus mutabilis, $\mathrm{HCH}$ ) sobre el rendimiento en crecimiento, desarrollo del tracto intestinal y valores hematológicos fueron evaluados en pavos machos de la línea BUT de un día de edad. Los pavos fueron distribuidos en cuatro grupos con dietas diferentes durante 56 días: dieta $\mathrm{L}_{0}$ (control) sin $\mathrm{HCH}$, y dietas $\mathrm{L}_{30}, \mathrm{~L}_{60} \mathrm{y} \mathrm{L}_{90}$ con 30,60 y $90 \mathrm{~g} / \mathrm{kg}$ de $\mathrm{HCH}$, respectivamente. El aumento de los niveles de inclusión de $\mathrm{HCH}$ en la dieta, agregados a expensas de la torta de soya, contribuyeron a un aumento en las concentraciones de fibra en las dietas experimentales. La ingesta diaria de alimento disminuyó en los tratamientos de $\mathrm{HCH}$ y la conversión alimenticia y el aumento de peso corporal de las aves se vieron afectados en tratamientos de 60 y $90 \mathrm{~g} / \mathrm{kg}$ de inclusión de $\mathrm{HCH}(\mathrm{p}<0.05)$. Los niveles dietéticos elevados de $\mathrm{HCH}$ llevaron a un aumento $(\mathrm{p}<0.01)$ de la digesta en el íleon y en el ciego, a un aumento en la masa relativa del tejido $(\mathrm{p}<0.01)$, así como una disminución en los valores de $\mathrm{pH}$ de la digesta $(\mathrm{p}<0.01)$. El incremento de $\mathrm{HCH}$ en la dieta no alteró los valores hematológicos. Se concluye que $\mathrm{HCH}$ puede considerarse como una alternativa segura y eficaz de inclusión dietética en niveles de $30 \mathrm{~g} / \mathrm{kg}$ en dietas de inicio para pavos jóvenes.

Palabras clave: semilla de chocho; productividad; desarrollo intestinal; valores hematológicos; pavos

\footnotetext{
${ }^{1}$ Departamento Académico de Ciencias Pecuarias, Facultad de Ingeniería en Ciencias Pecuarias, Universidad Nacional de Cajamarca, Perú

${ }^{2}$ E-mail:mepaunc@gmail.com,mparedes@unc.edu.pe
} 
The effect of four dietary levels of lupine seed meal (Lupinus mutabilis, HCH) on growth performance, intestinal tract development and haematological values were evaluated in male turkeys of the one-day BUT line. The turkeys were distributed in four groups with different diets for 56 days: $\mathrm{L}_{0}$ (control) diet without $\mathrm{HCH}$, and $\mathrm{L}_{30}, \mathrm{~L}_{60}$ and $\mathrm{L}_{90}$ diets containing 30,60 and $90 \mathrm{~g} / \mathrm{kg}$ of HCH, respectively. Increased levels of $\mathrm{HCH}$ inclusion in the diet, added at the expense of soybean meal, contributed to an increase in fibre concentrations in the experimental diets. Daily feed intake decreased in $\mathrm{HCH}$ treatments and feed conversion and bodyweight gain of birds were affected in treatments of 60 and $90 \mathrm{~g} / \mathrm{kg}$ of HCH inclusion $(\mathrm{p}<0.05)$. High dietary levels of HCH led to an increase $(\mathrm{p}<0.01)$ of the digesta in the ileum and in the cecum, an increase in the relative tissue mass $(\mathrm{p}<0.01)$, as well as a decrease in $\mathrm{pH}$ values of the digesta $(\mathrm{p}<0.01)$. The increase in $\mathrm{HCH}$ in the diet did not alter the haematological values. It is concluded that $\mathrm{HCH}$ can be considered as a safe and effective alternative for dietary inclusion at levels of $30 \mathrm{~g} / \mathrm{kg}$ in starting diets for young turkeys.

Key words: seed of lupine; productivity; intestinal development; hematological values; turkeys

\section{INTRODUCCIÓN}

El interés en el sector alimentario por identificar, producir y utilizar nuevas fuentes proteicas para la nutrición del ser humano y animales es una labor que no tiene término (Nalle et al., 2011). El sector avícola nacional y mundial buscan alternativas en este campo con el fin de incluir ingredientes que eficientemente reemplacen o superen a la torta de soya (TS), ingrediente proteico de mayor uso en la alimentación de aves, pues muchos países, como el Perú, no disponen de una producción industrial de esta semilla oleaginosa, dependiendo de su permanente importación.

En la sierra peruana existen diversos cultivos proteicos de la familia de las leguminosas, cultivados localmente, tales como como el chocho, conocido comúnmente como lupino, tarwi o altramuz. Estudios con semillas de lupino amarillo (Lupinus luteos) indican que se podría incluir en la dieta de pollos de engorde hasta en una proporción de 100/ $20 \mathrm{~g} / \mathrm{kg}$ (Farrell et al., 1999).
El principal factor limitante para el uso de semillas de lupino amarillo en la dieta de aves de corral es la alta concentración de polisacáridos no amiláceos (PNA), que reducen el valor nutricional de las semillas (van Barneveld, 1999), donde la rafinosa puede dificultar el transporte de nutrientes a través de la pared intestinal (Zdunczyk et al., 1998). El valor proteico de la semilla de Lupino mutabilis (LM) cosechada en la sierra peruana es similar al de semilla de soya, pero su utilización se ha limitado por otro factor adicional, como es la presencia de grandes cantidades de estructuras alcaloides quinolizidínicas, con cierto grado de toxicidad y sabor fuertemente amargo (Gutiérrez et al., 2016).

Otras especies y variedades de lupinos como el lupino amarillo, lupino blanco (Lupinus albus) o lupino azul (Lupinus angustifolius) han sido incluidas en las dietas finalizadoras de pavos de 18 semanas de edad en niveles de hasta $180 \mathrm{~g} / \mathrm{kg}$, sin que se afecte la función gastrointestinal y sin efectos adversos en el crecimiento o calidad de la carne (Mikulski et al., 2014), aun cuando 
puede causar aumento del peso de la molleja, disminución del $\mathrm{pH}$ de los contenidos de la molleja y problemas en la viscosidad de la digesta del intestino delgado (Zdunczyk et al., 2014). Por otro lado, Smulikowska et al. (2014) indicaron que las dietas de inicio con $100 \mathrm{~g} / \mathrm{kg}$ de semillas de lupino amarillo redujeron el peso corporal en pollos de engorde debido a una menor ingesta de alimento, además de cambios en el tracto gastrointestinal ocasionados por el aumento de las concentraciones de PNA y rafinosa.

La inclusión de LM en alimentación de pavos en sus primeras semanas de vida no está documentado. Así mismo, LM contiene más de 20 tipos de alcaloides o sustancias tóxicas que podrían provocar hemólisis y anemia (Rodríguez, 2009), como sucede con otros alcaloides vegetales cuyo efecto tóxico puede combinarse con el ion férrico en la sangre, afectando la capacidad de transporte de oxígeno de los eritrocitos; como ha sido demostrado en peces y patos jóvenes (Zeng et al., 2015). Actualmente, no existen reportes en pavos sobre el efecto de los alcaloides de LM sobre los valores hematológicos.

El presente estudio tuvo por objetivo determinar el efecto de diferentes niveles de inclusión dietética de harina de semilla de LM, agregado a expensas de TS, sobre el rendimiento productivo, desarrollo intestinal y valores hematológicos en pavos de 56 días de edad.

\section{Materiales y Métodos}

\section{Harina de Semilla de Chocho}

La semilla de chocho o tarwi (Lupino mutabilis - LM) cruda y sin ningún tratamiento se obtuvo de agricultores de la provincia de Cajamarca; Perú, y fue trasladada al Laboratorio de Control de Alimentos de la Universidad Nacional de Cajamarca, donde se llevó a cabo el proceso de eliminación de alcaloides o desamargado de la semilla de LM y realizar los análisis químicos.
La primera fase del procedimiento de desamargado de la semilla de LM concuerda con Gutiérrez et al. (2016), quienes remojaron la semilla en agua en una proporción de $1: 6$ durante $12 \mathrm{~h}$ a temperatura ambiente, para luego eliminar el agua de remojo y someter la semilla a cocción a $90{ }^{\circ} \mathrm{C}$ durante $60 \mathrm{~min}$, en una proporción de semilla: agua de 1:5. Esta operación se realizó en una olla programable de $10 \mathrm{~L}$ de capacidad. En una segunda fase se continuó con el proceso de lixiviación, colocando la semilla cocida en sacos de yute de $71 \times 101 \mathrm{~cm}$ a razón de $25 \mathrm{~kg}$ por saco, con una manguera conectada y suministro permanente de agua con un caudal de $21 / \mathrm{min}$ durante $24 \mathrm{~h}$. Luego se hizo la fase de secado a $80^{\circ} \mathrm{C}$ durante $48 \mathrm{~h}$ en una estufa para desecación XTemp de $80 \mathrm{~L}$ de capacidad. Finalmente, la semilla desecada fue triturada en un molino eléctrico con motor 3HP y tamiz de $2 \mathrm{~mm}$, obteniéndose la harina de semilla de chocho $(\mathrm{HCH})$.

La TS, la semilla cruda de LM y la $\mathrm{HCH}$ fueron analizados para materia seca (MS), proteína cruda $(\mathrm{PC})$, extracto etéreo (EE), fibra cruda (FC) y cenizas, utilizando los métodos AOAC (2005 correspondientes a $934.01,976.05,920.39,978.10$ y 942.05 , respectivamente. El extracto no nitrogenado (ENN) se obtuvo por diferencia. La composición química de la semilla cruda de LM y de la $\mathrm{HCH}$ se presentan en el Cuadro 1.

Cuadro 1. Composición química de la torta de soya (TS), semilla cruda de chocho (LM) y harina de chocho $(\mathrm{HCH})$ expresada en porcentaje y en base fresca

\begin{tabular}{lccc}
\hline Componentes & TS & LM & HCH \\
\hline Humedad & 11.4 & 11.5 & 12.1 \\
Proteína cruda & 44.5 & 45.1 & 41.2 \\
Extracto etéreo & 1.8 & 13.2 & 10.8 \\
Fibra cruda & 6.7 & 16.3 & 20.6 \\
Cenizas & 6.7 & 2.3 & 1.9 \\
Extracto no & 28.9 & 11.6 & 13.4 \\
nitrogenado & & & \\
\hline
\end{tabular}


Cuadro 2. Ingredientes y contenido nutricional de las ocho dietas experimentales $(\mathrm{g} / \mathrm{kg}$, base fresca)

\begin{tabular}{|c|c|c|c|c|c|c|c|c|}
\hline & \multicolumn{4}{|c|}{ Semana 0-4 } & \multicolumn{4}{|c|}{ Semana 4-8 } \\
\hline & $\mathrm{L}_{0}$ & $\mathrm{~L}_{30}$ & $\mathrm{~L}_{60}$ & $\mathrm{~L}_{90}$ & $\mathrm{~L}_{0}$ & $\mathrm{~L}_{30}$ & $\mathrm{~L}_{60}$ & $\mathrm{~L}_{90}$ \\
\hline Maíz & 395.0 & 395.0 & 395.0 & 395.0 & 440.0 & 440.0 & 440.0 & 440.0 \\
\hline Afrecho de trigo & 40.0 & 35.0 & 30.0 & 25.0 & 20.0 & 15.0 & 10.0 & 10.0 \\
\hline Harina de soya integral & - & 5.0 & 10.0 & 15.0 & 20.0 & 25.0 & 30.0 & 30.0 \\
\hline Torta de soya & 400.0 & 340.0 & 340.0 & 310.0 & 360.0 & 330.0 & 300.0 & 270.0 \\
\hline Harina de chocho & - & 30.0 & 60.0 & 90.0 & - & 30.0 & 60.0 & 90.0 \\
\hline Harina de pescado & 100.0 & 100.0 & 100.0 & 100.0 & 90.0 & 90.0 & 90.0 & 90.0 \\
\hline Aceite de soya & 15.0 & 15.0 & 15.0 & 15.0 & 20.0 & 20.0 & 20.0 & 20.0 \\
\hline Carbonato de calcio & 17.0 & 17.0 & 17.0 & 17.0 & 17.0 & 17.0 & 17.0 & 17.0 \\
\hline Fosfato dicálcico & 19.5 & 19.5 & 19.5 & 19.5 & 19.5 & 19.5 & 19.5 & 19.5 \\
\hline Sal & 2.5 & 2.5 & 2.5 & 2.5 & 2.5 & 2.5 & 2.5 & 2.5 \\
\hline Bicarbonato de sodio & 1.0 & 1.0 & 1.0 & 1.0 & 1.0 & 1.0 & 1.0 & 1.0 \\
\hline DL metionina & 3.0 & 3.0 & 3.0 & 3.0 & 3.0 & 3.0 & 3.0 & 3.0 \\
\hline Lisina HCL & 3.0 & 3.0 & 3.0 & 3.0 & 3.0 & 3.0 & 3.0 & 3.0 \\
\hline Premezcla vitamínica mineral $^{\mathrm{a}}$ & 1.0 & 1.0 & 1.0 & 1.0 & 1.0 & 1.0 & 1.0 & 1.0 \\
\hline Aditivos $^{\mathrm{b}}$ & 3.0 & 3.0 & 3.0 & 3.0 & 3.0 & 3.0 & 3.0 & 3.0 \\
\hline \multicolumn{9}{|l|}{$\begin{array}{l}\text { Contenido de nutrientes } \\
\text { calculado }\end{array}$} \\
\hline Materia seca & 895.2 & 896.4 & 897.7 & 898.9 & 895.9 & 897.2 & 898.5 & 899.7 \\
\hline Proteína cruda & 275.6 & 275.7 & 275.9 & 276 & 260 & 260 & 260.2 & 259.2 \\
\hline $\begin{array}{l}\text { Energía metabolizable, } \\
\mathrm{kcal} / \mathrm{kg}\end{array}$ & 2744 & 2748 & 2752 & 2755 & 2842 & 2845 & 2849 & 2846 \\
\hline Fibra cruda & 43.6 & 47.2 & 50.8 & 54.4 & 40.1 & 43.7 & 47.3 & 51.3 \\
\hline Lisina & 19.7 & 19.7 & 19.6 & 19.6 & 18.5 & 18.5 & 18.5 & 18.3 \\
\hline Metionina & 8.5 & 8.5 & 8.5 & 8.5 & 8.2 & 8.2 & 8.2 & 8.2 \\
\hline Calcio & 14.6 & 14.6 & 14.6 & 14.6 & 14 & 14 & 14 & 14 \\
\hline Fósforo disponible & 7.9 & 7.9 & 7.9 & 7.9 & 7.6 & 7.6 & 7.6 & 7.6 \\
\hline
\end{tabular}

$\mathrm{L}_{0}, \mathrm{~L}_{30}, \mathrm{~L}_{60}$ y $\mathrm{L}_{90}: 0,30,60,90 \mathrm{~g} / \mathrm{kg}$ de harina de semilla de chocho $(\mathrm{HCH})$ en la dieta, respectivamente, como sustituto parcial de la torta de soya

${ }^{a}$ Cada kg contiene: Vit. A 10000 mil UI, Vit. D3 3000 mil UI, Vit. E 12000 UI, Vit. K3 2.5 g, tiamina 2 g, riboflavina $6 \mathrm{~g}$, cianocobalamina $12 \mathrm{mg}$, ácido pantoténico $16 \mathrm{~g}$, ácido fólico $21.5 \mathrm{~g}$, niacina $120 \mathrm{mg}$, Mn 65 g, Zn 65 g, Fe 80 g, Cu 10 g, I 1 g, Se 200 mg

${ }^{\mathrm{b}}$ La mezcla de aditivos contiene: Fungiban (ácido propiónico al 99.5\%) $1 \mathrm{~g}$, bacitrazinc (bacitracina zinc al 15\%) $0.5 \mathrm{~g}$, Nitarpro (nitarsona al 20\%) 1g, Uniban (Dinitro ortho toluamida al 25\%) $0.5 \mathrm{~g}$

\section{Dietas}

Las ocho dietas experimentales se muestran en el Cuadro 2. Las dietas en cada fase alimenticia fueron isonitrogenadas $(27.5 \mathrm{y}$ $26 \%$ de PC en fases de 1 a 4 y de 5 a 8 semanas, respectivamente) e isocalóricas (2800 y $2900 \mathrm{kcal} / \mathrm{kg}$ de EM para las dos fases). La EM de la $\mathrm{HCH}$ fue calculada de acuerdo con la ecuación $\mathrm{EM}=4.31 \mathrm{~g}$ de $\mathrm{PC}$ digestible $+9.29 \mathrm{~g}$ de EE digestible $+4.14 \mathrm{~g}$ de ENN digestible (Rostagno et al., 2005). Los valores de $0.80,0.50$ y $0.27 \mathrm{de}$ digestibilidad para la PC, EE y ENN de $\mathrm{HCH}$, respectivamente, fueron considerados como referenciales (Rostagno et al., 2005) para la torta de soya. Todas las dietas cumplieron o excedieron las especificaciones del NRC (1994) referentes a vitaminas y minerales. La TS de origen boliviano, importada por la 
empresa ContiLatin Perú fue adquirida de un abastecedor minorista de la ciudad de Trujillo, molida y envasada en sacos de $50 \mathrm{~kg}$.

\section{Aves y Alojamiento}

Se trabajó con 400 pavos de la línea BUT (British United Turkey), variedad BIG6 , de un día de edad, procedentes de la Corporación Gramobier, Lima-Perú. Fueron alojados en el galpón de aves de la Universidad Nacional de Cajamarca, y asignados al azar en cuatro tratamientos dietéticos, cada uno con cinco repeticiones y cada repetición con 20 aves por corral.

Las aves se criaron en piso con cama de viruta y se proporcionó 24 horas de luz durante la primera semana y 16 horas de luz por día en las siguientes semanas. La temperatura ambiente se mantuvo a $32{ }^{\circ} \mathrm{C}$ durante los primeros 7 días y se disminuyó gradualmente en $2{ }^{\circ} \mathrm{C}$ por semana hasta llegar a $22^{\circ} \mathrm{C}$ a la sexta semana, luego de lo cual los pavos se mantuvieron a temperatura ambiente. Las aves tuvieron acceso libre a dietas tipo harina, administradas a lo largo de los dos periodos alimenticios de cuatro semanas cada uno (1-4 y 5-8). Las aves se vacunaron a los 7 días utilizando Newcastle B1B1 + Bronquitis Mass, a los 14 días con Poulvac TRT contra rinotraqueítis, a los 21 días con Viruela poxine y a los 28 días con Newcastle LS + Bronquitis Mass (Montana, Perú).

\section{Parámetros Productivos}

El peso corporal (PC), ganancia de peso corporal (GPC) y consumo de alimento fueron registrados y calculados sobre la base de las aves existentes en un corral, cada cuatro semanas (28 y 56 días). La ingesta diaria de alimento (IDA) por ave se calculó sobre la base del consumo total de alimento de cada corral para todo el periodo experimental y para el número de días por cada fase alimen- ticia. La conversión alimenticia (CA) para el periodo y por cada fase alimenticia se calculó sobre la base de la ganancia de peso corporal y el consumo de alimento, considerando la relación de kilogramos de alimento consumido sobre kilogramos de GPC. Para medir el PC y el consumo de alimento se utilizó una balanza de precisión KERN EW 6000 $1 \mathrm{M}$ (Alemania), de capacidad $6000 \mathrm{~g}$ y precisión de lectura $0.1 \mathrm{~g}$.

\section{Desarrollo Intestinal}

A las ocho semanas de alimentación se sacrificaron dos aves por corral por dislocación cervical de acuerdo con las recomendaciones para el sacrificio de animales experimentales (Close et al., 1997). Los segmentos del tracto digestivo (intestino delgado y ciego) se extrajeron, se vaciaron y se pesaron. A los 20 min, se midió el pH ileal y cecal utilizando un microelectrodo y un medidor de iones de $\mathrm{pH}$, modelo $780 \mathrm{pH}$ Meter, Metrohm International Headquarters. Se usaron muestras de contenido ileal y cecal para el análisis inmediato de MS. Los ciegos e íleon se lavaron con solución salina fisiológica, se secaron sobre papel filtro y se pesaron. El protocolo de pesado de intestinos y digesta fue según Jankowski et al. (2009).

\section{Análisis de Sangre}

Se recolectaron $2 \mathrm{ml}$ de sangre de la vena braquial de cada ave, cumplidos los 56 días de edad, a las 07:00 del día, considerando la muestra de sangre de un ave como una repetición, y se colocaron en tubos que contenían EDTA-Na ${ }_{2}$ para la determinación de los valores hematológicos: hematocrito (HCT, $\%$ ), hemoglobina (HGB, g/l), recuento de leucocitos $\left(\mathrm{L}, \mathrm{miles} / \mathrm{mm}^{3}\right)$ y el recuento de glóbulos rojos $\left(\mathrm{GR} \times 10^{2} / 1\right)$. El análisis se realizó en el Laboratorio Regional del Norte, Cajamarca, utilizando un analizador de hematología automatizado (Alfa Basic 16p, Boule Medical AB, Suecia) dentro de las $2 \mathrm{~h}$ posteriores a la extracción de sangre. 


\section{Análisis Estadístico}

Se utilizó el análisis de varianza para determinar los efectos de los niveles dietéticos de $\mathrm{HCH}$ sobre los parámetros de productividad, desarrollo intestinal y valores hematológicos mediante el procedimiento GLM del software SAS (SAS, 2006). Las diferencias significativas entre los grupos se determinaron mediante la prueba de rango múltiple de Duncan. Las diferencias se consideraron significativas a $\mathrm{p}<0.05$.

\section{Resultados y Discusión}

\section{Parámetros Productivos}

Las dietas experimentales fueron isonitrogenadas reemplazando parte de TS con HCH (Cuadro 2). Así mismo, se consideraron pequeñas cantidades de harina integral de soya en las fórmulas alimenticias para nivelar las densidades de energía y proteína de las dietas, proporcionalmente al aumento del contenido de $\mathrm{HCH}$. También se añadió afrecho de trigo, en cantidades inversas a la inclusión de $\mathrm{HCH}$, con el propósito de disminuir las diferencias en el contenido de FC de los piensos, sin lograr tal propósito. Así, las concentraciones de FC fueron las más bajas en el alimento $L_{0}$, y se incrementaron con el aumento de las tasas de inclusión de $\mathrm{HCH}$ en las dietas experimentales y, por tanto, se incrementó también el contenido de PNA en estas dietas, por ser la celulosa y la hemicelulosa de la FC componentes de los PNA (FEDNA, 2018).

El contenido de FC fue aproximadamente un $25 \%$ mayor en la dieta $\mathrm{L}_{90}$, en comparación con la dieta $\mathrm{L}_{0}$. Durante la alimentación experimental de 56 días, las tasas de mortalidad no difirieron entre los grupos, siendo en cada tratamiento de $2 \%$. Durante ese periodo, la IDA disminuyó ( $\mathrm{p}=0.05$ ) a medida que aumentaban los niveles de $\mathrm{HCH}$ en la dieta, con una disminución alta de la IDA en las primeras cuatro semanas de alimentación experimental $(p<0.01)$. Los pavos control y los alimentados con dietas que contenían $30 \mathrm{~g} / \mathrm{kg} \mathrm{HCH}$ se caracterizaron por una GPC similar (3.30 y $3.19 \mathrm{~kg}$ en el periodo de 56 días) mientras que la GPC de los pavos alimentados con dietas que contenían 60 y $90 \mathrm{~g} / \mathrm{kg}$ $\mathrm{HCH}$ fueron más bajos que los valores del grupo control $(\mathrm{p}=0.048)$. Durante la primera fase alimenticia se observaron cambios similares en GPC $(p<0.05)$, al igual que en la segunda fase $(\mathrm{p}=0.021)$. La CA se afectó $(\mathrm{p}<0.01)$ con el aumento del contenido de $\mathrm{HCH}$ en las dietas (Cuadro 3).

Los pavos alimentados con dietas con 60 y 90 g/ $/ \mathrm{kg}$ de contenido de $\mathrm{HCH}$ se caracterizaron por un menor GPC, en particular cuando se compara con el grupo control. Ese efecto coincide con la disminución de la eficiencia alimenticia en los tratamientos de $\mathrm{HCH}$, cuyas concentraciones de fibra fueron considerablemente más altas en $\mathrm{HCH}$ que en TS. Este efecto dietético coincide con lo demostrado por Santos et al. (2006), quienes determinaron que cantidades excesivas de fibra dietética afectan negativamente la digestibilidad de los nutrientes y reducen el rendimiento del crecimiento de aves de engorde. Sin embargo, en otros estudios, la inclusión de $240 \mathrm{~g} / \mathrm{kg}$ de semillas de lupino amarillo a una dieta con gran cantidad de fibra con pavos de 9 a 16 semanas no tuvo una influencia negativa en los parámetros de rendimiento de las aves o la calidad de la carne (Krawczyk et al., 2015). Al parecer la cantidad de fibra de las dietas con $\mathrm{HCH}$ excedió los niveles mínimos indicados por Mateos et al. (2012), quienes afirman que, la adición moderada de componentes altos en fibra a las dietas de cereales modula positivamente la función gastrointestinal en aves.

Los resultados de este estudio difieren a los de Zdunczyc et al. (2016), quienes evaluaron pavos de ocho semanas y encontraron mejor GPC con $160 \mathrm{~g} / \mathrm{kg}$ de lupino amarillo en la dieta y mejor conversión alimenticia con $240 \mathrm{~g} / \mathrm{kg}$ del mismo lupino, atribuyendo los mejores parámetros productivos al mayor contenido de grasa; sin embargo, en el presente estudio, aun cuando el LM tiene 
Cuadro 3. Ganancia de peso corporal (GPC), ingesta diaria de alimento (IDA) y conversión alimenticia (CA) de pavos de ocho semanas alimentados con dietas conteniendo cuatro niveles de harina de semilla de chocho (Lupino mutabilis) ${ }^{1}$

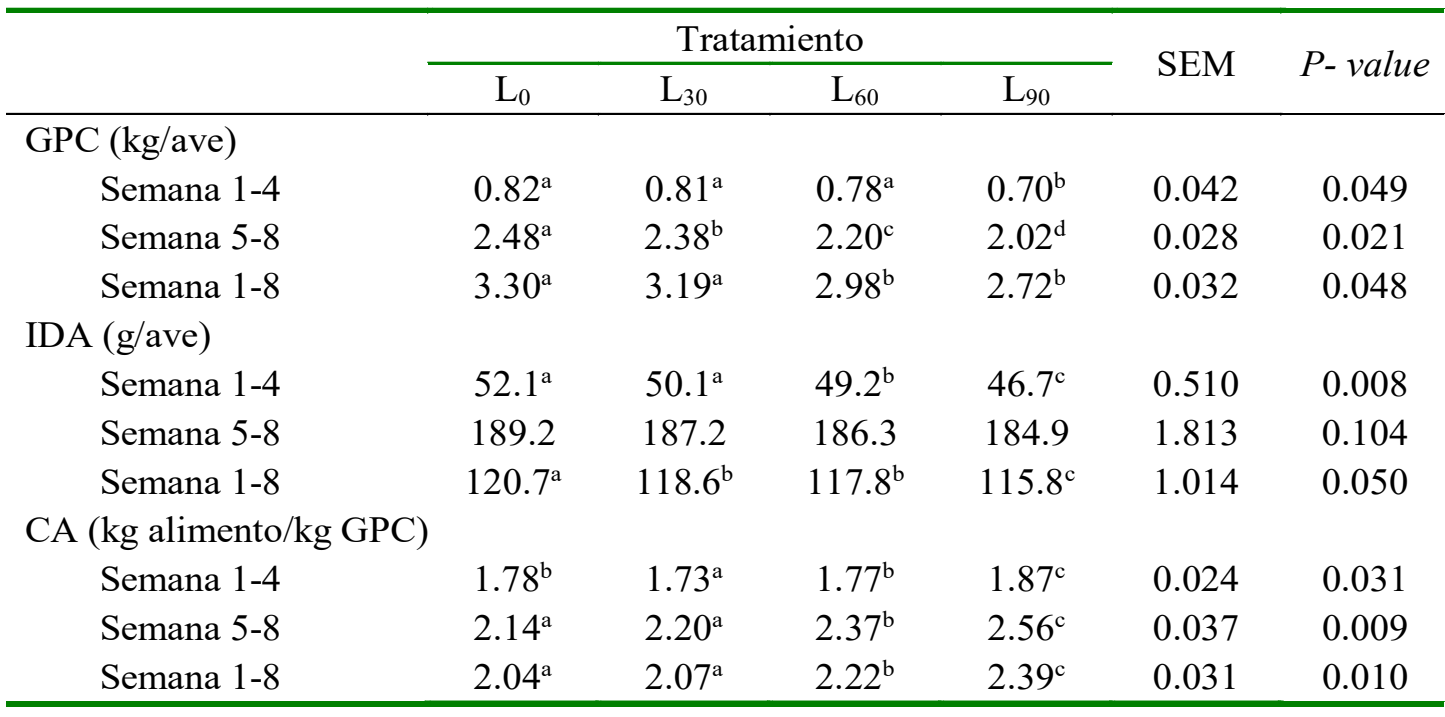

${ }^{1}$ Los datos representan promedios de cinco repeticiones por tratamiento de 20 pavos por repetición $L_{0}, L_{30}, L_{60}$ y $L_{90}: 0,30,60,90 \mathrm{~g} / \mathrm{kg}$ de harina de semilla de chocho $(\mathrm{HCH})$ en la dieta, respectivamente, como sustituto parcial de la torta de soya

SEM: Error estándar de la media

$\mathrm{a}, \mathrm{b}, \mathrm{c}, \mathrm{d}$ Promedios en la misma fila con diferentes superíndices son estadísticamente diferentes $(p<0.05)$

alto contenido de EE, posiblemente su mayor componente fibroso no corresponda al tipo de fibra dietética, que es la que aumenta la secreción de ácidos biliares y el contenido de alfaamilasa del quimo y facilita la emulsificación de los lípidos (Hetland y Svihus, 2001).

\section{Desarrollo Intestinal}

La incorporación de $\mathrm{HCH}$ en las dietas aumentó la masa tisular cecal relativa ( $p=0.012$; Cuadro 4). Un aumento en el contenido de $\mathrm{HCH}$ en las dietas condujo a una disminución en el $\mathrm{pH}$ cecal ( $\mathrm{p}=0.011)$, acompañado por un aumento en las concentraciones de MS $(\mathrm{p}<0.01)$.

No se observaron cambios en el $\mathrm{pH}$ de la digesta ileal, lo que sugiere que $\mathrm{HCH}$ no estimuló la fermentación en el intestino del- gado. Resultados similares se han encontrado en otros experimentos con pavos de hasta 18 semanas de edad (Mikulski et al., 2014). La inclusión de $\mathrm{HCH}$ en las dietas para pavos aumentó la masa relativa del tejido cecal y el contenido de materia seca de la digesta cecal, y disminuyó el $\mathrm{pH}$ cecal, debido a que la HCH contribuyó, posiblemente, a un aumento de las concentraciones de PNA y rafinosa en las dietas para pavos, estimulando la actividad de enzimas glucolíticas de la microbiota cecal (Zdunczyk et al., 2016). Esos cambios, indican que la $\mathrm{HCH}$ en la dieta afectó los procesos de fermentación del ecosistema cecal de pavos en crecimiento, lo que coincide con procesos de fermentación en el ciego encontrados en otros estudios en pavos y pollos de engorde alimentados con dietas suplementadas con lupino (Mikulski et al., 2014). 
Cuadro 4. Parámetros de desarrollo intestinal de pavos de ocho semanas alimentados con dietas conteniendo cuatro niveles de harina de semilla de chocho (Lupino mutabilis $)^{1}$

\begin{tabular}{|c|c|c|c|c|c|c|}
\hline & \multicolumn{4}{|c|}{ Tratamiento } & \multirow{2}{*}{ SEM } & \multirow{2}{*}{ P-value } \\
\hline & $\mathrm{L}_{0}$ & $\mathrm{~L}_{30}$ & $\mathrm{~L}_{60}$ & $\mathrm{~L}_{90}$ & & \\
\hline $\begin{array}{l}\text { Peso del tejido de } \\
\text { intestino delgado, } \\
\text { g/kg de PV }\end{array}$ & 24.1 & 24.7 & 25.8 & 26.7 & 0.478 & 0.313 \\
\hline $\begin{array}{l}\text { Peso de la digesta del } \\
\text { intestino delgado, } \\
\text { g/kg de PV }\end{array}$ & 26.3 & 26.4 & 27.9 & 30.3 & 0.691 & 0.224 \\
\hline $\begin{array}{r}\text { Materia seca de la } \\
\text { digesta ileal, \% }\end{array}$ & 12.8 & 13.7 & 13.1 & 13.2 & 0.943 & 0.871 \\
\hline $\mathrm{pH}$ de la digesta ileal & 6.09 & 6.2 & 6.31 & 6.39 & 0.702 & 0.239 \\
\hline $\begin{array}{l}\text { Peso del tejido cecal, } \\
\text { g/kg de PV }\end{array}$ & $5.12^{\mathrm{b}}$ & $5.87^{\mathrm{a}}$ & $5.99^{\mathrm{a}}$ & $6.27^{\mathrm{a}}$ & 0.714 & 0.012 \\
\hline $\begin{array}{l}\text { Peso de la digesta cecal, } \\
\text { g/kg de PV }\end{array}$ & 4.33 & 4.38 & 4.39 & 4.4 & 0.671 & 0.779 \\
\hline $\begin{array}{l}\text { Materia seca de la } \\
\text { digesta cecal, \% }\end{array}$ & $15.8^{\mathrm{c}}$ & $15.9^{\mathrm{c}}$ & $16.8^{\mathrm{b}}$ & $17.4^{\mathrm{a}}$ & 1.722 & 0.008 \\
\hline $\mathrm{pH}$ de la digesta cecal & $6.41^{\mathrm{a}}$ & $6.17^{\mathrm{b}}$ & $6.02^{\mathrm{b}}$ & $5.94^{\mathrm{b}}$ & 0.738 & 0.011 \\
\hline
\end{tabular}

Cuadro 5. Valores hematológicos de pavos de ocho semanas de edad alimentados con dietas conteniendo diferentes niveles de harina de semilla de chocho (Lupino mutabilis) ${ }^{1}$

\begin{tabular}{|c|c|c|c|c|c|c|}
\hline & \multicolumn{4}{|c|}{ Tratamiento } & \multirow{2}{*}{ SEM } & \multirow{2}{*}{$P$-value } \\
\hline & $\mathrm{L}_{0}$ & $\mathrm{~L}_{30}$ & $\mathrm{~L}_{60}$ & $\mathrm{~L}_{90}$ & & \\
\hline Eritrocitos (x 100/ml) & 2.65 & 3.00 & 2.76 & 2.83 & 0.09 & 0.09 \\
\hline Leucocitos $(1000 / \mathrm{ml})$ & 6.30 & 6.45 & 6.40 & 6.50 & 0.11 & 0.42 \\
\hline Hemoglobina $(\mathrm{g} / 100 \mathrm{ml})$ & 11.8 & 11.5 & 11.9 & 11.2 & 0.20 & 0.25 \\
\hline Hematocrito $(\%)$ & 30.2 & 29.7 & 30.3 & 30.3 & 0.94 & 0.75 \\
\hline Linfocitos (\%) & 38 & 38 & 37 & 35 & 1.83 & 0.41 \\
\hline
\end{tabular}

${ }^{1}$ Datos representan promedios de cinco repeticiones por tratamiento de un pavo por repetición $L_{0}, L_{30}, L_{60}$ y $L_{90}: 0,30,60,90 \mathrm{~g} / \mathrm{kg}$ de harina de semilla de chocho $(\mathrm{HCH})$ en la dieta, respectivamente, como sustituto parcial de la torta de soya

SEM: Error estándar de la media 


\section{Valores Hematológicos}

El aumento del nivel de uso de $\mathrm{HCH}$ en la dieta no afectó los valores de hematológicos del pavo a los 56 días de edad (Cuadro 5).

En general, la actividad citotóxica de los alcaloides se asocia con una disminución de la concentración de hemoglobina y hematocrito, y desarrollo de anemia (Berardi y Goldblatt, 1980). En el presente estudio no se encontraron tales anomalías, coincidiendo con Torres (2006) quien administró un extracto de fitoquímico de chocho conteniendo lupanina, esparteína y demás alcaloides a ovejas sin encontrar alteraciones de los valores normales en sangre. Sin embargo, se debe tener en cuenta que los alcaloides contenidos en el chocho o lupino presentan valores $\mathrm{DL}_{50}$ de lupanina más altos que la esparteína (Cubillos et al., 1999).

\section{Conclusiones}

La inclusión de harina de semilla de chocho (Lupino mutabilis) $(\mathrm{HCH})$ en niveles de $30 \mathrm{~g} / \mathrm{kg}$ en reemplazo de esa misma cantidad de torta de soya en la dieta para pavos en crecimiento durante las primeras ocho semanas de edad, produce un efecto beneficioso sobre los parámetros productivos y el desarrollo intestinal, en comparación a mayores niveles de inclusión dietaria de $\mathrm{HCH}$.

\section{Literatura Citada}

1. AOAC. 2005. Official Methods of Analysis of the Association of the Official Analytical Chemists. $18^{\text {th }}$ ed. AOAC International, Arlington, USA.

2. Berardi LC, Goldblatt LA. 1980. Gossypol. In: Toxic constituents of plant feedstuff. New York, USA: Academic Press. p 184- 237.

3. Close B, Banister K, Baumans V, Bernoth EM, Bromage N, Bunyan J, Erhardt W, et al. 1997. Recommen- dations for euthanasia of experimental animals: Part 2. Lab Animal 31: 1-32. doi: 10.1258/002367797780-600297

4. Cubillos A, Gädicke P, von Baer D, Ahumada F. 1999. Determinación de la dosis letal media $\left(\mathrm{DL}_{50}\right)$ de alcaloides del lupino en pollas de reposición blancas y marón. Arch Med Vet 31: 249-256. doi: 10.4067/S0301-732X1999000200014

5. Farrell DJ, Perez-Maldanado RA, Mannion PF. 1999. Optimum inclusion of field peas, faba beans, chick peas and sweet lupins in poultry diets. II. Broiler experiments. Brit Poultry Sci 40: 674680. doi: 10.1080/00071669987061

6. [FEDNA] Fundación Española para el Desarrollo de la Nutrición Animal. 2018. Necesidades nutricionales para avicultura. España. 194 p. ]Internet]. Disponible en: http://www.fundacion fedna.org/sites/default/files/ NORMAS_FEDNA_AVES_2018v.pdf

7. Gutiérrez A, Infantes M, Pascual G, Zamora J. 2016. Evaluación de los factores en el desamargado de tarwi (Lupinus mutabilis Sweet). Agroind Sci 6: 145-149.

8. Hetland H, Svihus B. 2001. Effect of oat hulls on performance, gut capacity and feed passage time in broiler chickens. Brit Poultry Sci 42: 354-361. doi: 10.1080/ 00071660120055331

9. Jankowski J, Juskiewicz, J, Gulewicz K, Lecewicz A, Slominski BA, Zdunczyk $Z$. 2009. The effect of diets containing soybean meal, soybean protein concentrate, and soybean protein isolate of different oligosaccharide content on growth performance and gut function of young turkeys. J Poult Sci 88: 2132-2140. doi: 10.3382/ps.2009-00066

10. Krawczyk M, Mikulski D, Przywitowski M, Jankowski J. 2015. The effect of dietary yellow lupine (L. Luteus cv. Baryt) on growth performance, carcass characteristics, meat quality and selected serum parameters of turkeys. J Anim Feed Sci 24: 61-70. doi: 10.22358/ jafs $/ 65654 / 2015$ 
11. Mateos GG, Jiménez-Moreno E, Serrano MP, Lázaro RP. 2012. Poultry response to high levels of dietary fiber sources varying in physical and chemical characteristics. J Appl Poultry Res 21: 156-174. doi: 10.3382/japr.2011-00477

12. Mikulski D, Zdunczyk Z, Juskiewicz J, Rogiewicz A, Jankowski J. 2014. The effect of different blue lupine ( $L$. angustifolius) inclusion levels on gastrointestinal function, growth performance and meat quality in growingfinishing turkeys. Anim Feed Sci Tech 198: 347-352. doi: 10.1016/j.anifeedsci. 2014.10.005

13. Nalle CL, Ravindran $\mathrm{V}$, Ravindran $\boldsymbol{G}$ 2011. Nutritional value of narrow-leafed lupin (Lupinus angustifolius) for broi-lers. Brit Poultry Sci 52: 775-81. doi: 10.1080/ 00071668.2011.639343

14. [NRC] National Research Council. 1994. Nutrient requirements of poultry. $9^{\text {th }}$ ed. Washington, DC: National Academies Press.

15. Rodríguez A. 2009. Evaluación «in vitro» de la actividad antimicrobiana de los alcaloides del agua de cocción del proceso de desamargado del chocho (Lupinus mutabilis Sweet). Tesis de Bioquímico Farmacéutico. Riobamba, Ecuador: Escuela Superior Politécnica de Chimborazo. $83 \mathrm{p}$.

16. Rostagno HR, Teixeira LF, Donzele JL, Gomez, PC, de Oliveira RF, Lopes, DC, Ferreira, AS, et al. 2005. Tablas brasileñas para aves y cerdos. Composición de alimentos y requerimientos nutricionales. $2^{\circ}$ ed. Brasil: Univ. Federal de Viçosa. 186 p.

17. SAS Institute Inc. 2006. SAS/STAT User's guide. Release 9.1. SAS Institute, Inc., Cary, NC.

18. Santos FBO, Santos AA, Ferket PR, Seldon $B W$. 2006. Influence of grain particle size and insoluble fiber content on Salmonella colonization and shedding of turkeys fed corn-soybean meal diets. Int J Poult Sci 5: 731-739. doi: 10.3923/ ijps.2006.731.739

19. Smulikowska S, Konieczka P, Czerwinski J, Mieczkowska A, Jankowiak J. 2014. Feeding broiler chickens with particle diets containing lupin seeds $(L$. angustifolius or L. luteus): effect of incorporation level and mannanase supplementation on growth performance, digesta viscosity, microbial fermentation and gut morphology. J Anim Feed Sci 23: 64-72.

20. Torres L. 2006. Utilización del chocho (Lupinis mutabilis Sweet) como antiparasitario gastrointestinal y hepático en ovinos mestizos. Tesis de Ingeniero Zootecnista. Ecuador: Escuela Superior Politécnica de Chimborazo. $90 \mathrm{p}$.

21. van Barneveld RJ. 1999. Understanding the nutritional chemistry of lupin (Lupinus spp.) seeds to improve livestock production efficiency. Nutr Res Rev 12: 203-30. doi: 10.1079/095442299108728938

22. Zdunczyk Z, Juskiewicz J, Frejnagel S, Gulewicz, K. 1998. Influence of alkaloids and oligosaccharides from white lupin seeds on utilization of diets by rats and absorption of nutrients in the small intestine. Anim Feed Sci Tech 72: 143-154. doi: 10.1016/S0377-8401(97)00173-9

23. Zdunczyk Z, Jankowski J, Mikulski D, Mikulska M, Lamparski G, Slominski BA. Juskiewicz J. 2014. Growth performance, gastrointestinal function and meat quality in growingfinishing turkeys fed diets with different levels of yellow lupine (L. luteus) seeds. Arch Anim Nutr 68: 211-226.

24. Zdunczyk Z, Krawczyk M, Mikulski D, Jankowski J, PrzybylskaGornowiczc B, Juskiewicz J. 2016. Beneficial effects of increasing dietary levels of yellow lupine (Lupinus luteus) seed meal on productivity parameters and gastrointestinal tract physiology in eight-week-old turkeys. Anim Feed Sci Tech 211: 189-198. doi: 10.1016/ j.anifeedsci.2015.11.015

25. Zeng QF, Bai P, Wang JP, Ding XM, Luo YH, Bai SP, Xuan Y, Su ZW, Lin SQ, Zhao LJ, Zhang KY. 2015. The response of meat ducks from 15 to $35 \mathrm{~d}$ of age to gossypol from cottonseed meal. Poultry Sci 94: 1277-1286. doi: 10.3382/ ps/pev070 